\title{
Allylation of Functionalized Aldehydes by Potassium Allyltrifluoroborate Catalyzed by 18-Crown-6 in Aqueous Media
}

\author{
Fernanda C. G. Barbosa, Juliano C. R. Freitas, Caio F. Melo, Paulo H. Menezes and \\ Roberta A. Oliveira * \\ Departamento de Química Fundamental, Universidade Federal de Pernambuco, \\ Av. Jornalista Aníbal Fernandes, s/n - Cidade Universitária, Recife - PE, 50740-560, Brazil \\ * Author to whom correspondence should be addressed; E-Mail: roberta.aoliveira@ufpe.br; \\ Tel.: +55-81-2126-7473; Fax: +55-81-2126-8442.
}

Received: 25 September 2012; in revised form: 19 November 2012 / Accepted: 23 November 2012 / Published: 28 November 2012

\begin{abstract}
An efficient method for the allylation of aldehydes containing a broad range of functional groups using potassium allyltrifluoroborate is described. The reaction utilizes a catalytic amount of 18-C-6 in biphasic media under open atmosphere and room temperature to provide the corresponding homoallylic alcohols in high yields and without the necessity of any subsequent purification.
\end{abstract}

Keywords: potassium allyltrifluoroborate; allylation; 18-crown-6

\section{Introduction}

The addition of allylic organometallics to aldehydes is an important method for C-C bond formation [1], while the product obtained from this reaction, an homoallylic alcohol, is an analogue of the compound obtained from the aldol addition of metal enolates [2]. In addition, the introduced alkene moiety can be readily converted into the corresponding aldehyde for further carbon-carbon homologation; or can be selectively epoxidized to introduce a new chiral center [3].

The general approaches to prepare homoallylic alcohols are based on the use of an allylic organometallic (e.g., Li [4], Mg [5], Mn [6], Zn [7], Pb [8], Bi [9], Ce [10], In [11], Sn [12] and many others [13-17]), organometalloid derivatives (e.g., B [18-20], Si [21]) and electrochemical based methods [22]. However, in some cases, these reagents are expensive and difficult to handle due to their Lewis base character. Alternative methods based on the Barbier reaction were also developed [23,24]. 
These methods avoid the previous preparation of the allylic organometallics, however, the use of zero valent metals can cause metal oxide (or hydroxide) precipitation and Rieke metals and some chlorosilanes are also sensitive to the reaction media.

Organoboron compounds have proved to be very useful in the allylation of carbonyl compounds because of their easy formation and stability and to date, several methods for the allylation of carbonyl compounds employing allyl boronic acids or boronic esters were developed [18-20].

Potassium allyltrifluoroborates have proven to be a good option to replace allylboronic acids and allylboronate esters in allylation reactions, providing many advantages over the latter reagents [25-28]. These compounds are stable, easy to handle, compatible with several functional groups and can be stored for a long time. Several methods for the allylation of carbonyl compounds using allylic trifluoroborates promoted by a variety of Lewis acids [29-31] or palladium-catalysts [32] have been described. However, the search for an easy and reliable method for the allylation of aldehydes, that could be performed in an open flask with substrates containing a broad range of functional groups would be desirable, while some of the previously described methods for the allylation of aldehydes present drawbacks such as the price of catalysts [33] and the need to use anhydrous conditions [34].

In addition, the development of methods focusing on environmentally benign reaction media has become particularly prominent [35,36] and the use of water as (co)-solvent, which would seem to be the best option due to its simplicity and very low cost, is still a challenge.

Crown ethers have the ability to promote reactions by phase-transfer catalysis solubilizing inorganic salts in apolar solvents. Because of their remarkable binding properties, many reactions can be promoted by using these compounds as catalysts. In addition, the study of crown ethers has greatly contributed to the development of host-guest chemistry and supramolecular chemistry [37].

Herein, we wish to describe an environmentally benign reaction for the synthesis of homoallylic alcohols based on the reaction of potassium allyltrifluoroborate and aldehydes containing different functional groups in aqueous media catalyzed by 18 -crown-6.

\section{Results and Discussion}

Because our initial studies were focused on the development of an optimum set of reaction conditions, the reaction of 4-nitrobenzaldehyde (1a) and potassium allyltrifluoroborate (2) was examined to optimize these conditions. Therefore, $\mathbf{1 a}(1 \mathrm{mmol})$ and $\mathbf{2}(1.2 \mathrm{mmol})$ were treated at room temperature with the appropriate catalyst $\left(10 \mathrm{~mol} \%\right.$ ) using different $\mathrm{CH}_{2} \mathrm{Cl}_{2}-\mathrm{H}_{2} \mathrm{O}$ ratios, and the progress of the reaction was monitored by TLC. The results are presented in Table 1 (all reported yields in this and other tables are isolated yields).

When a 1:1 mixture of $\mathrm{CH}_{2} \mathrm{Cl}_{2}-\mathrm{H}_{2} \mathrm{O}$ was used as the reaction solvent, product 3a was obtained in good yields, even when the amount of the catalyst was decreased (Table 1, entries 2 and 3). However, with the variation in the amount of $18-C-6$, longer reaction times were required to promote the reaction (Table 2, entries 1 and 2). Further decreasing in the amount of catalyst required even longer reaction times and gave a lower yield. 
Table 1. Effect of solvent and catalyst on the allylation of 4-nitrobenzaldehyde (1a) by potassium allyltrifluoroborate 2 .

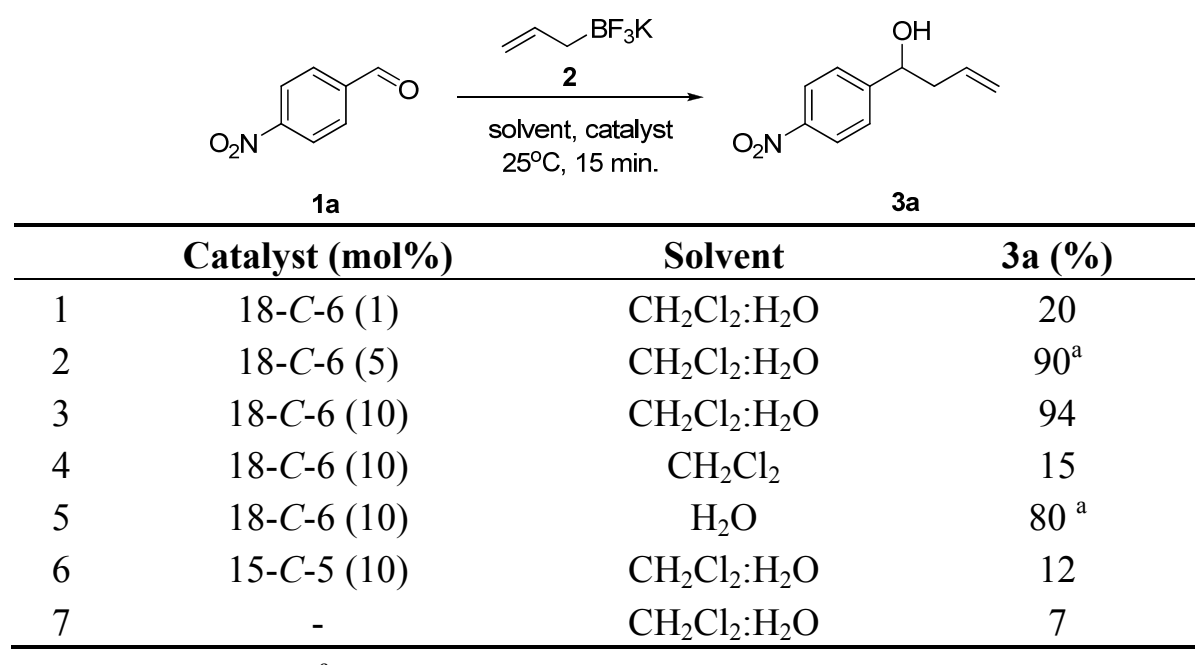

${ }^{a}$ Yield obtained after $30 \mathrm{~min}$ of reaction.

Table 2. Allylation of aldehydes $\mathbf{1}$ with potassium allyl trifluoroborate $\mathbf{2}$ catalyzed by $18-C-6(10 \mathrm{~mol} \%)$.

$$
\mathrm{R}_{\mathbf{1}} \frac{\begin{array}{c}
18-\mathrm{C}-6(10 \mathrm{~mol} \%) \\
\mathrm{CH}_{2} \mathrm{Cl}_{2}: \mathrm{H}_{2} \mathrm{O}(1: 1) \\
25^{\circ} \mathrm{C}, 15 \mathrm{~min} .
\end{array}}{\mathrm{BF}_{3} \mathrm{~K}}
$$

(\%)


Table 2. Cont.

(\%)

When only $\mathrm{CH}_{2} \mathrm{Cl}_{2}$ was used as the reaction solvent, lower yields were obtained in the allylation reaction, probably due to the low solubility of 2 in this solvent (Table 1, entry 4). A similar behavior was observed when only water was used as the reaction solvent, again due to the low solubility of the aldehyde in water (Table 1, entry 5). In the absence of $18-C-6$, the product was obtained only in $7 \%$ yield after 15 min (Table 1, entry 7) and when 15-C-5 was used as catalyst, lower yields were observed (Table 1, entry 6). This fact may be explained by the role of cation solvation by the ligand in size match-selectivity phenomena, which is widely accepted as the most important factor in controlling metal ion selectivity for macrocyclic ligands.

With the optimized reaction conditions, we next explored the generality of our method, by extending the conditions to different aldehydes. The results are listed in Table 2. In all cases, the reaction took place smoothly to give the corresponding homoallylic alcohols in good yields.

Various differently substituted aldehydes were used in the reaction. The allylation of aldehydes containing other electron-withdrawing groups gave the corresponding products in high yields (Table 2, entries 1-6). For example, when 2-, 3- and 4-nitrobenzaldehyde were used, similar yields were observed, indicating that the substituent position has a little influence in the reaction (Table 2, entries 1-3). Aldehydes containing electron-withdrawing groups such as 4-fluorobenzaldehyde (Table 2, entry 4); 4-bromobenzaldehyde (Table 2, entry 5) and 4-chlorobenzaldehyde (Table 2, entry 6) also gave similar yields. Other aromatic aldehydes such as $\beta$-naphtaldehyde (Table 2, entry 9), benzaldehyde (Table 2, entry 10) and 4-methylbenzaldehyde (Table 2, entry 11) also gave the homoallylic alcohols in high yields. When an $\alpha, \beta$-unsaturated aldehyde was used, the corresponding 1,2-addition product was obtained exclusively, proving that the reaction is regioselective (Table 2, entry 8). For aliphatic aldehydes, the 18-C-6-catalyzed allylation also exhibited high efficiency (Table 2, entry 7). Electron-rich aldehydes reacted in good yields without influence of the substituent location (Table 2, entries 12-14). When a heterocyclic aldehyde reacted under the optimized conditions, the corresponding homoallylic 
alcohol was obtained in good yield (Table 2, entry 15). We attribute the small differences in the isolated yields to the different solubilities of reactants and products.

As we can see, this new method has several advantages in comparison to other previously described approaches. First of all, some methods are based on the use of allyl organometallics. It is known that these compounds are sometimes not suitable for carbonyl additions due to the deprotonation of the acidic alpha protons. Because of that the use of nonenolizable aldehydes is required. In addition, to avoid the Wurtz coupling, the preparation of allylic Grignard reagents requires low temperature and concentration [38].

The use of less reactive compounds such as allylsilanes [21] and allylstannanes [12] requires Lewis acids as additives. Although the utility of allylstannanes is further indicated by the commercial availability of some of them, the toxicity of these compounds makes them inappropriate for the use in pharmaceutical synthesis [39]. In addition, the removal of tributyltin residues from reaction mixtures is also a major issue.

On the other hand, among the various allylboron reagents, the use of potassium allyltrifluoroborate seems to be the best option due to its stability, which also allows the complete characterization of these salts by heteronuclear NMR analysis [40], and exact mass measurements [41]. Additionally, organotrifluoroborate salts appear to have low toxicity [42].

Previously described methods for the allylation of aldehydes based on the conversion of potassium allyltrifluoroborate into the corresponding air and moisture sensitive allyl difluoroborane using $\mathrm{BF}_{3} \cdot \mathrm{Et}_{2} \mathrm{O}$ [34], or palladium catalysts [32] were reported. However, the use of potassium allyltrifluoroborate as the allylating agent without any previous transformation is an important advantage since it can be used in aqueous media and open atmosphere.

Furthermore, the use of potassium $(E)$-crotyltrifluoroborate (4) gave selectively the corresponding anti product 5 in a 96:4 anti:syn ratio, proving that the reaction was also diastereoselective (Scheme 1).

Scheme 1. Crotylation of 4-nitrobenzaldehyde.<smiles>O=Cc1ccc([N+](=O)[O-])cc1</smiles>

$1 \mathrm{a}$

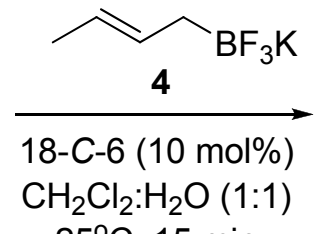

$25^{\circ} \mathrm{C}, 15 \mathrm{~min}$.

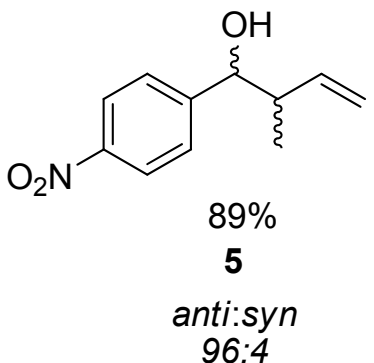

96:4

Finally, to exemplify the utility of the proposed method in the synthesis of natural products, we applied it to the synthesis of C7-C17 fragment of (-)-macrolactin F [43,44], a 24-membered macrolactone isolated from a marine Bacillus sp. Sc026 which exhibited antibacterial activity against Bacillus subtilis and Staphylococcus aureus (Scheme 2). 
Scheme 2. Synthesis of C7-C17 fragment of (-)-macrolactin F.

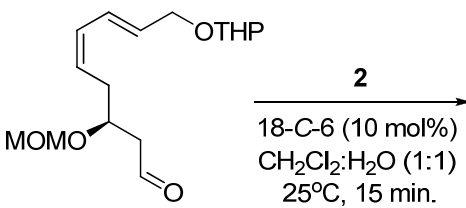

6
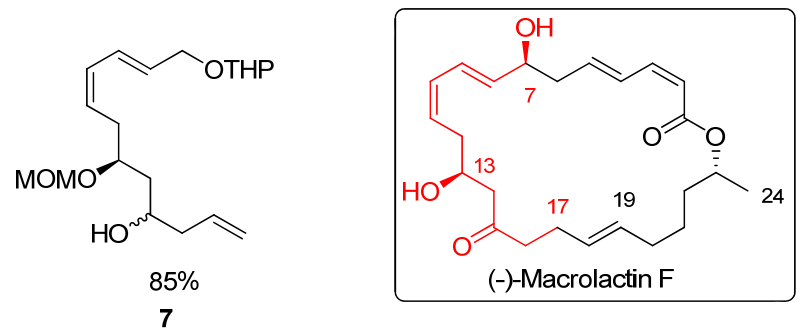

\section{Experimental}

\subsection{General}

${ }^{1} \mathrm{H}-\mathrm{NMR}$ and ${ }^{13} \mathrm{C}$-NMR data were recorded in $\mathrm{CDCl}_{3}$ on a Varian Unity Plus 300 spectrometer. The chemical shifts are reported as delta $(\delta)$ units in parts per million $(\mathrm{ppm})$ relative to the solvent residual peak as the internal reference. Reactions were monitored by thin-layer chromatography on $0.25 \mathrm{~mm} \mathrm{E}$. Merck silica gel 60 plates (F254) using UV light, vanillin and $p$-anisaldehyde as visualizing agents. Potassium allyltrifluoroborate [45] and potassium (E)-crotyltrifluoroborate [34] were prepared according to the literature procedures.

\subsection{General Procedure for the Allylation of Aldehydes 1a-0 with Potassium Allyltrifluoroborate (2)}

Catalyzed by 18-C-6

To a solution of the appropriate aldehyde $1 \mathbf{a}-\mathbf{0}(1.0 \mathrm{mmol})$ in $\mathrm{CH}_{2} \mathrm{Cl}_{2}(2 \mathrm{~mL})$ was added 18-C-6 (26 mg, $10 \mathrm{~mol} \%$ ) followed by potassium allyltrifluoroborate (2, $177 \mathrm{mg}, 1.20 \mathrm{mmol}$ ) and water $(2 \mathrm{~mL})$. The biphasic mixture was stirred for 15 minutes, diluted with EtOAc $(15 \mathrm{~mL})$ and washed with a saturated solution of $\mathrm{K}_{2} \mathrm{CO}_{3}(3 \times 15 \mathrm{~mL})$. The combined organic layers were dried over $\mathrm{MgSO}_{4}$, filtered and the solvents were removed in vacuo to yield $\mathbf{3 a - 0}$ [46] without the need of further purification.

1-(4-Nitrophenyl)but-3-en-1-ol (3a): Yield: $181 \mathrm{mg}$ (94\%); ${ }^{1} \mathrm{H}-\mathrm{NMR}\left(\mathrm{CDCl}_{3}\right) \delta 8.20(\mathrm{~d}, J=8.7 \mathrm{~Hz}$, $\left.2 \mathrm{H}, \mathrm{H}_{\text {Aryl }}\right), 7.53\left(\mathrm{~d}, J=8.7 \mathrm{~Hz}, 2 \mathrm{H}, \mathrm{H}_{\text {Aryl }}\right), 5.85-5.71\left(\mathrm{~m}, 1 \mathrm{H}, \mathrm{CH}=\mathrm{CH}_{2}\right), 5.22-5.16\left(\mathrm{~m}, 2 \mathrm{H}, \mathrm{CH}=\mathrm{CH}_{2}\right)$, $4.86(\mathrm{dd}, J=7.8,4.5 \mathrm{~Hz}, 1 \mathrm{H}, \mathrm{CHOH}), 2.61-2.39\left(\mathrm{~m}, 2 \mathrm{H}, \mathrm{CHCH}_{2}\right), 2.15$ (br s, $\left.1 \mathrm{H}, \mathrm{OH}\right) ;{ }^{13} \mathrm{C}-\mathrm{NMR}$ $\left(\mathrm{CDCl}_{3}\right) \delta 151.1,147.1,133.1,126.5,123.5,119.5,72.1,43.8$.

1-(3-Nitrophenyl)but-3-en-1-ol (3b): Yield: $154 \mathrm{mg}(82 \%)$; ${ }^{1} \mathrm{H}-\mathrm{NMR}\left(\mathrm{CDCl}_{3}\right) \delta 8.23(\mathrm{t}, J=1.5 \mathrm{~Hz}, 1 \mathrm{H}$, $\left.\mathrm{H}_{\text {Aryl }}\right), 8.12$ (ddd $\left.J=8.1,2.1,0,9 \mathrm{~Hz}, 1 \mathrm{H}, \mathrm{H}_{\text {Aryl }}\right), 7.69$ (d, $\left.J=8.1 \mathrm{~Hz}, 1 \mathrm{H}, \mathrm{H}_{\text {Aryl }}\right), 7.53$ (t, $J=8.1 \mathrm{~Hz}, 1 \mathrm{H}$, $\left.\mathrm{H}_{\text {Aryl }}\right), 5.86-5.72\left(\mathrm{~m}, 1 \mathrm{H}, \mathrm{CH}=\mathrm{CH}_{2}\right), 5.21-5.15\left(\mathrm{~m}, 2 \mathrm{H}, \mathrm{CH}=\mathrm{CH}_{2}\right), 4.86(\mathrm{dd}, J=8.1,5.1 \mathrm{~Hz}, 1 \mathrm{H}$, $\mathrm{CHOH}), 2.62-2.42\left(\mathrm{~m}, 2 \mathrm{H}, \mathrm{CHCH}_{2}\right), 2.17$ (br s, $\left.1 \mathrm{H}, \mathrm{OH}\right) ;{ }^{13} \mathrm{C}-\mathrm{NMR}\left(\mathrm{CDCl}_{3}\right) \delta 148.1,145.9,133.2$, $131.9,129.3,122.4,120.8,119.6,72.0,43.9$.

1-(2-Nitrophenyl)but-3-en-1-ol (3c): Yield: $158 \mathrm{mg}(80 \%) ;{ }^{1} \mathrm{H}-\mathrm{NMR}\left(\mathrm{CDCl}_{3}\right) \delta 7.92(\mathrm{dd}, J=8.1,1.2 \mathrm{~Hz}$, $\left.1 \mathrm{H}, \mathrm{H}_{\text {Aryl }}\right), 7.83\left(\mathrm{dd}, J=8.1,1.5 \mathrm{~Hz}, 1 \mathrm{H}, \mathrm{H}_{\text {Aryl }}\right), 7.67$ (td, $\left.J=8.1,1.2 \mathrm{~Hz}, 1 \mathrm{H}, \mathrm{H}_{\text {Aryl }}\right), 7.42$ (td, $J=8.1$, $\left.1.2 \mathrm{~Hz}, 1 \mathrm{H}, \mathrm{H}_{\text {Aryl }}\right), 5.96-5.82\left(\mathrm{~m}, 1 \mathrm{H}, \mathrm{CH}=\mathrm{CH}_{2}\right), 5.31$ (dd, $\left.J=8.4,3.6 \mathrm{~Hz}, 1 \mathrm{H}, \mathrm{CHOH}\right), 5.23-5.17$ $\left(\mathrm{m}, 2 \mathrm{H}, \mathrm{CH}=\mathrm{CH}_{2}\right), 2.75-2.66\left(\mathrm{~m}, 1 \mathrm{H}, \mathrm{CHCH}_{2}\right), 2.47-2.36\left(\mathrm{~m}, 2 \mathrm{H}, \mathrm{CHCH}_{2}\right.$ and $\left.\mathrm{OH}\right)$; ${ }^{13} \mathrm{C}-\mathrm{NMR}$ $\left(\mathrm{CDCl}_{3}\right) \delta 147.7,139.2,133.9,133.4,128.1,128.0,124.3,119.0,68.3,42.8$. 
1-(4-Fluorophenyl)but-3-en-1-ol (3d): Yield: $144 \mathrm{mg}(87 \%)$; ${ }^{1} \mathrm{H}-\mathrm{NMR}\left(\mathrm{CDCl}_{3}\right) \delta$ 7.28-7.24 (m, 2H, $\left.\mathrm{H}_{\text {Aryl }}\right), 6.96\left(\mathrm{t}, 2 \mathrm{H}, J=8.4 \mathrm{~Hz}, \mathrm{H}_{\text {Aryl }}\right), 5.79-5.65\left(\mathrm{~m}, 1 \mathrm{H}, \mathrm{CH}=\mathrm{CH}_{2}\right), 5.12-5.07\left(\mathrm{~m}, 2 \mathrm{H}, \mathrm{CH}=\mathrm{CH}_{2}\right), 4.66$ (dd, $1 \mathrm{H}, J=7.2,5.7 \mathrm{~Hz}, \mathrm{CHOH}), 2.50-2.38\left(\mathrm{~m}, 2 \mathrm{H}, \mathrm{CHCH}_{2}\right), 1.85$ (br s, $\left.1 \mathrm{H}, \mathrm{OH}\right) ;{ }^{13} \mathrm{C}-\mathrm{NMR} \delta$ $\left(\mathrm{CDCl}_{3}\right) \delta 164.1,139.2,133.8,127.1,118.4,114.9,72.3,43.6$.

1-(4-Chlorophenyl)but-3-en-1-ol (3e): Yield: $155 \mathrm{mg}$ (85\%); ${ }^{1} \mathrm{H}-\mathrm{NMR}\left(\mathrm{CDCl}_{3}\right) \delta$ 7.27-7.19 (m, 4H, $\left.\mathrm{H}_{\text {Aryl }}\right), 5.79-5.62\left(\mathrm{~m}, 1 \mathrm{H}, \mathrm{CH}=\mathrm{CH}_{2}\right), 5.13-5.05\left(\mathrm{~m}, 2 \mathrm{H}, \mathrm{CH}=\mathrm{CH}_{2}\right), 4.65$ (dd, $\left.J=7.5,5.4 \mathrm{~Hz}, \mathrm{CHOH}\right)$, 2.48-2.32 (m, 2H, $\left.\mathrm{CHCH}_{2}\right), 2.04$ (br s, $\left.1 \mathrm{H}, \mathrm{OH}\right) ;{ }^{13} \mathrm{C}-\mathrm{NMR}\left(\mathrm{CDCl}_{3}\right) \delta 142.2,133.9,131.1,128.5$, $127.2,118.8,72.5,43.8$.

1-(4-Bromophenyl)but-3-en-1-ol (3f): Yield: $194 \mathrm{mg}(86 \%) ;{ }^{1} \mathrm{H}-\mathrm{NMR}\left(\mathrm{CDCl}_{3}\right) \delta 7.41(\mathrm{~d}, J=8.7 \mathrm{~Hz}$, $\left.2 \mathrm{H}, \mathrm{H}_{\text {Aryl }}\right), 7.17\left(\mathrm{~d}, J=8.7 \mathrm{~Hz}, 2 \mathrm{H}, \mathrm{H}_{\text {Aryl }}\right), 5.78-5.64\left(\mathrm{~m}, 1 \mathrm{H}, \mathrm{CH}=\mathrm{CH}_{2}\right), 5.13-5.06\left(\mathrm{~m}, 2 \mathrm{H}, \mathrm{CH}=\mathrm{CH}_{2}\right)$, $4.64(\mathrm{dd}, J=7.8,5.4 \mathrm{~Hz}, \mathrm{CHOH}), 2.49-2.32\left(\mathrm{~m}, 2 \mathrm{H}, \mathrm{CHCH}_{2}\right), 1.99$ (br s, $\left.1 \mathrm{H}, \mathrm{OH}\right) ;{ }^{13} \mathrm{C}-\mathrm{NMR}\left(\mathrm{CDCl}_{3}\right) \delta$ $142.8,133.9,131.4,127.5,121.2,118.9,72.5,43.8$.

Non-1-en-4-ol (3g): Yield: $126 \mathrm{mg}(89 \%) ;{ }^{1} \mathrm{H}-\mathrm{NMR}\left(\mathrm{CDCl}_{3}\right) \delta$ 5.89-5.76 (m, 1H, $\left.\mathrm{CH}=\mathrm{CH}_{2}\right), 5.29-5.09$ $\left(\mathrm{m}, 2 \mathrm{H}, \mathrm{CH}=\mathrm{CH}_{2}\right), 3.68-3.59(\mathrm{~m}, 1 \mathrm{H}, \mathrm{CHOH}), 2.34-2.25\left(\mathrm{~m}, 2 \mathrm{H}, \mathrm{CHCH}_{2}\right), 2.18-2.07(\mathrm{~m}, 2 \mathrm{H}$, $\mathrm{CHOH}) 1.66$ (br s, $1 \mathrm{H}, \mathrm{OH}), 150-1.25\left(\mathrm{~m}, 6 \mathrm{H}, \mathrm{CH}_{3} \mathrm{CH}_{2} \mathrm{CH}_{2} \mathrm{CH}_{2}\right), 0.87$ (t, $J=6.3 \mathrm{~Hz}, 6 \mathrm{H}, \mathrm{CH}_{3} \mathrm{CH}_{2}$ ); ${ }^{13} \mathrm{C}-\mathrm{NMR}\left(\mathrm{CDCl}_{3}\right) \delta 134.9,118.0,70.6,41.9,36.7,31.8,25.3,22.6,14.0$.

(E)-1-Phenylhexa-1,5-dien-3-ol (3h): Yield: $157 \mathrm{mg}(90 \%) ;{ }^{1} \mathrm{H}-\mathrm{NMR}\left(\mathrm{CDCl}_{3}\right) \delta 7.40-7.21\left(\mathrm{~m}, 5 \mathrm{H}, \mathrm{H}_{\text {Aryl }}\right)$, $6.61(\mathrm{dd}, J=15.9,1.2 \mathrm{~Hz}, 1 \mathrm{H}, \mathrm{PhCH}=\mathrm{CH}), 6.24(\mathrm{dd}, J=15.9,6.3 \mathrm{~Hz}, 1 \mathrm{H}, \mathrm{PhCH}=\mathrm{CH}), 5.86$ (ddt, $\left.J=17.1,10.2,6.9 \mathrm{~Hz}, 1 \mathrm{H}, \mathrm{CH}=\mathrm{CH}_{2}\right), 5.22-5.14\left(\mathrm{~m}, 2 \mathrm{H}, \mathrm{CH}=\mathrm{CH}_{2}\right), 4.39-4.33(\mathrm{~m}, 1 \mathrm{H}, \mathrm{CHOH})$, 2.50-2.34 (m, 2H, $\left.\mathrm{CHCH}_{2}\right), 1.78$ (br s, $\left.1 \mathrm{H}, \mathrm{OH}\right) ;{ }^{13} \mathrm{C}-\mathrm{NMR}\left(\mathrm{CDCl}_{3}\right) \delta 136.5,133.9,131.5,130.2$, $128.5,127.6,126.4,118.3,71.6,41.9$.

1-(Naphthalen-2-yl)but-3-en-1-ol (3i): Yield: $172 \mathrm{mg}(87 \%) ;{ }^{1} \mathrm{H}-\mathrm{NMR}\left(\mathrm{CDCl}_{3}\right) \delta$ 7.86-7.81 (m, 4H, $\left.\mathrm{H}_{\text {Aryl }}\right), 7.53-7.47$ (m, 3H, $\left.\mathrm{H}_{\text {Aryl }}\right), 5.84$ (ddt, $\left.J=17.1,10.2,7.5 \mathrm{~Hz}, 1 \mathrm{H}, \mathrm{CH}=\mathrm{CH}_{2}\right), 5.23-5.14(\mathrm{~m}, 2 \mathrm{H}$, $\left.\mathrm{CH}=\mathrm{CH}_{2}\right), 4.91(\mathrm{dd}, J=7.2,5.1 \mathrm{~Hz}, 1 \mathrm{H}, \mathrm{CHOH}), 2.61-2.57\left(\mathrm{~m}, 2 \mathrm{H}, \mathrm{CHCH}_{2}\right), 2.10$ (br s, $\left.1 \mathrm{H}, \mathrm{OH}\right)$; ${ }^{13} \mathrm{C}-\mathrm{NMR}\left(\mathrm{CDCl}_{3}\right) \delta 141.2,134.3,133.2,132.9,128.1,127.9,127.6,126.1,125.8,124.2,123.9,118.4$, 73.3, 43.6 .

1-Phenyl-but-3-en-1-ol (3j): Yield: $130 \mathrm{mg}(88 \%) ;{ }^{1} \mathrm{H}-\mathrm{NMR}\left(\mathrm{CDCl}_{3}\right) \delta 7.36-7.24\left(\mathrm{~m}, 5 \mathrm{H}, \mathrm{H}_{\text {Aryl }}\right)$, 5.87-5.73 (m, 1H, $\left.\mathrm{CH}=\mathrm{CH}_{2}\right), 5.19-5.11\left(\mathrm{~m}, 2 \mathrm{H}, \mathrm{CH}=\mathrm{CH}_{2}\right), 4.73(\mathrm{dd}, J=7.5,5.4 \mathrm{~Hz}, 1 \mathrm{H}, \mathrm{CHOH})$, 2.54-2.47 (m, 2H, $\left.\mathrm{CHCH}_{2}\right), 2.00($ br s, $1 \mathrm{H}, \mathrm{OH}) ;{ }^{13} \mathrm{C}-\mathrm{NMR} \delta\left(\mathrm{CDCl}_{3}\right) \delta 143.8,134.4,128.2,127.3$, $125.7,118.0,73.2,43.6$.

1-p-Tolyl-but-3-en-1-ol (3k): Yield: $133 \mathrm{mg}(82 \%) ;{ }^{1} \mathrm{H}-\mathrm{NMR}\left(\mathrm{CDCl}_{3}\right) \delta 7.22\left(\mathrm{~d}, J=7.8 \mathrm{~Hz}, 2 \mathrm{H}, \mathrm{H}_{\text {Aryl }}\right)$, $7.16\left(\mathrm{~d}, J=7.8 \mathrm{~Hz}, 2 \mathrm{H}, \mathrm{H}_{\text {Aryl }}\right), 5.81$ (ddd, $\left.1 \mathrm{H}, J=17.1,10.2,6.6 \mathrm{~Hz}, \mathrm{CH}=\mathrm{CH}_{2}\right), 5.20-5.11(\mathrm{~m}, 2 \mathrm{H}$, $\left.\mathrm{CH}=\mathrm{CH}_{2}\right), 4.71(\mathrm{t}, J=6.6 \mathrm{~Hz}, \mathrm{CHOH}), 2.56-2.48\left(\mathrm{~m}, 2 \mathrm{H}, \mathrm{CHCH}_{2}\right), 2.35\left(\mathrm{~s}, 3 \mathrm{H}, \mathrm{CH}_{3}\right), 1.78($ br s, $1 \mathrm{H}$, $\mathrm{OH}) ;{ }^{13} \mathrm{C}-\mathrm{NMR}\left(\mathrm{CDCl}_{3}\right) \delta 140.9,137.2,134.6,129.0,125.7,118.2,73.1,43.7,21.1$.

1-(4-Methoxyphenyl)but-3-en-1-ol (3I): Yield: $158 \mathrm{mg}(89 \%) ;{ }^{1} \mathrm{H}-\mathrm{NMR}\left(\mathrm{CDCl}_{3}\right) \delta 7.77(\mathrm{~d}, J=9.0 \mathrm{~Hz}$, $\left.2 \mathrm{H}, \mathrm{H}_{\text {Aryl }}\right), 7.37$ (d, $\left.J=9.0 \mathrm{~Hz}, 2 \mathrm{H}, \mathrm{H}_{\text {Aryl }}\right), 6.29$ (ddt, $\left.J=16.8,9.9,6.6 \mathrm{~Hz}, 1 \mathrm{H}, \mathrm{CH}=\mathrm{CH}_{2}\right), 5.68-5.59$ 
(m, 2H, CH=CH2), 5.17 (t, $J=6.6 \mathrm{~Hz}, \mathrm{CHOH}), 4.29$ (s, 3H, OMe), 3.01-2.96 (m, 2H, $\left.\mathrm{CHCH}_{2}\right), 2.50$ (br s, $1 \mathrm{H}, \mathrm{OH}) ;{ }^{13} \mathrm{C}-\mathrm{NMR}\left(\mathrm{CDCl}_{3}\right) \delta 158.9,136.0,134.6,127.0,118.2,113.7,72.9,55.2,43.7$.

1-(3-Methoxyphenyl)but-3-en-1-ol (3m): Yield: $160 \mathrm{mg}(90 \%) ;{ }^{1} \mathrm{H}-\mathrm{NMR}\left(\mathrm{CDCl}_{3}\right) \delta 7.29(\mathrm{dd}, J=8.1$, $\left.7.8 \mathrm{~Hz}, 1 \mathrm{H}, \mathrm{H}_{\text {Aryl }}\right), 6.97-6.94\left(\mathrm{~m}, 2 \mathrm{H}, \mathrm{H}_{\text {Aryl }}\right), 6.84$ (ddd, $J=8.1,2.7,1.2 \mathrm{~Hz}, 1 \mathrm{H}, \mathrm{H}_{\text {Aryl }}$ ), 5.84 (ddt, $\left.J=17.1,10.2,7.5 \mathrm{~Hz}, 1 \mathrm{H}, \mathrm{CH}=\mathrm{CH}_{2}\right), 5.23-5.15\left(\mathrm{~m}, 2 \mathrm{H}, \mathrm{CH}=\mathrm{CH}_{2}\right), 4.74(\mathrm{dd}, J=7.5,5.4 \mathrm{~Hz}, 1 \mathrm{H}$, $\mathrm{CHOH}), 3.84(\mathrm{~s}, 3 \mathrm{H}, \mathrm{OMe}), 2.56-2.50\left(\mathrm{~m}, 2 \mathrm{H}, \mathrm{CHCH}_{2}\right), 2.06(\mathrm{br} \mathrm{s}, 1 \mathrm{H}, \mathrm{OH}),{ }^{13} \mathrm{C}-\mathrm{NMR}\left(\mathrm{CDCl}_{3}\right) \delta$ $159.6,145.6,134.4,129.3,118.2,118.0,112.9,111.2,73.1,55.1,43.6$.

1-(2-Methoxyphenyl)but-3-en-1-ol (3n): Yield: $162 \mathrm{mg}(91 \%) ;{ }^{1} \mathrm{H}-\mathrm{NMR}\left(\mathrm{CDCl}_{3}\right) \delta 7.33(\mathrm{dd}, J=7.5$, $\left.1.8 \mathrm{~Hz}, 1 \mathrm{H}, \mathrm{H}_{\text {Aryl }}\right), 7.28\left(\mathrm{td}, J=7.5,1.8 \mathrm{~Hz}, 1 \mathrm{H}, \mathrm{H}_{\text {Aryl }}\right), 6.95\left(\mathrm{td}, J=8.4,1.2 \mathrm{~Hz}, 1 \mathrm{H}, \mathrm{H}_{\text {Aryl }}\right), 6.87$ (d, $\left.J=8.4 \mathrm{~Hz}, 1 \mathrm{H}, \mathrm{H}_{\text {Aryl }}\right), 5.85\left(\mathrm{ddt}, J=17.1,10.2,7.5 \mathrm{~Hz}, 1 \mathrm{H}, \mathrm{CH}=\mathrm{CH}_{2}\right), 5.17-5.08\left(\mathrm{~m}, 2 \mathrm{H}, \mathrm{CH}=\mathrm{CH}_{2}\right)$, $4.95(\mathrm{dd}, J=8.1,5.1 \mathrm{~Hz}, 1 \mathrm{H}, \mathrm{CHOH}), 3.84$ (s, 3H, OMe), 2.64-2.44 (m, 2H, $\left.\mathrm{CHCH}_{2}\right), 2.41$ (br s, $1 \mathrm{H}$, $\mathrm{OH}) ;{ }^{13} \mathrm{C}-\mathrm{NMR}\left(\mathrm{CDCl}_{3}\right) \delta 156.2,135.1,131.7,128.2,126.7,120.6,117.4,110.3,69.5,55.1,41.8$.

1-(Furan-2-ylbut-3-en-1-ol (3o): Yield: $119 \mathrm{mg}(86 \%) ;{ }^{1} \mathrm{H}-\mathrm{NMR}\left(\mathrm{CDCl}_{3}\right) \delta 7.38(\mathrm{dd}, J=1.8,0.9 \mathrm{~Hz}$, $\left.1 \mathrm{H}, \mathrm{H}_{\mathrm{Het}}\right), 6.33$ (dd, $\left.J=2.1,1.8 \mathrm{~Hz}, 1 \mathrm{H}, \mathrm{H}_{\mathrm{Het}}\right), 6.25$ (dd, $\left.J=2.1,0.9 \mathrm{~Hz}, 1 \mathrm{H}, \mathrm{H}_{\mathrm{Het}}\right), 5.81$ (ddt, $J=17.1$, $\left.10.2,6.9 \mathrm{~Hz}, 1 \mathrm{H}, \mathrm{CH}=\mathrm{CH}_{2}\right), 5.23-5.13\left(\mathrm{~m}, 2 \mathrm{H}, \mathrm{CH}=\mathrm{CH}_{2}\right), 4.75(\mathrm{dd}, J=6.6,6.3 \mathrm{~Hz}, 1 \mathrm{H}, \mathrm{CHOH})$, 2.66-2.60 (m, 2H, $\left.\mathrm{CHCH}_{2}\right), 2.15$ (br s, $\left.1 \mathrm{H}, \mathrm{OH}\right) ;{ }^{13} \mathrm{C}-\mathrm{NMR}\left(\mathrm{CDCl}_{3}\right) \delta 155.6 ; 141.9 ; 133.6,118.6$, $110.1,106.1,66.9,40.1$.

\subsection{General Procedure for the Crotylation of 1a with Potassium (E)-crotyltrifluoroborate (4)}

Catalyzed by 18-C-6

To a solution of aldehyde $1 \mathrm{a}(38 \mathrm{mg}, 0.25 \mathrm{mmol})$ in $\mathrm{CH}_{2} \mathrm{Cl}_{2}(1 \mathrm{~mL})$ was added 18-C-6 (6.5 mg, $10 \mathrm{~mol} \%$ ) followed by potassium $(E)$-crotyltrifluoroborate $(4,49 \mathrm{mg}, 0.3 \mathrm{mmol})$ and water $(1 \mathrm{~mL})$. The biphasic mixture was stirred for $15 \mathrm{~min}$, diluted with EtOAc $(15 \mathrm{~mL})$ and washed with a saturated solution of $\mathrm{K}_{2} \mathrm{CO}_{3}(3 \times 10 \mathrm{~mL})$. The combined organic layers were dried over $\mathrm{MgSO}_{4}$, filtered and the solvents were removed in vacuo to yield 5 [47] without the need of further purification.

2-Methyl-1-(4-nitrophenyl)but-3-en-1-ol (5): Yield: $55 \mathrm{mg}(89 \%) ;{ }^{1} \mathrm{H}-\mathrm{NMR}\left(\mathrm{CDCl}_{3}\right) \delta 8.21(\mathrm{~d}, J=8.7 \mathrm{~Hz}$, $\left.2 \mathrm{H}, \mathrm{H}_{\text {Aryl }}\right), 7.51$ (d, $\left.J=8.7 \mathrm{~Hz}, 2 \mathrm{H}, \mathrm{H}_{\text {Aryl }}\right), 5.75$ (ddd, $J=16.8,10.5,8.1 \mathrm{~Hz}, 1 \mathrm{H}, \mathrm{CH}=\mathrm{CH}_{2}$ ), 5.23-5.15 $\left(\mathrm{m}, 2 \mathrm{H}, \mathrm{CH}=\mathrm{CH}_{2}\right), 4.52(\mathrm{~d}, J=7.2 \mathrm{~Hz}, 1 \mathrm{H}, \mathrm{CHOH}), 2.47$ (sex, $\left.J=7.2 \mathrm{~Hz}, 2 \mathrm{H}, \mathrm{CHCH}\right), 2.17$ (br s, 1H, $\mathrm{OH}), 0.94\left(\mathrm{~d}, J=7.2 \mathrm{~Hz}, 3 \mathrm{H}, \mathrm{CH}_{3}\right) ;{ }^{13} \mathrm{C}-\mathrm{NMR}\left(\mathrm{CDCl}_{3}\right) \delta 15.0,139.4,127.8,123.4,118.1,76.9,46.5$, 25.0, 16.5 .

\subsection{General Procedure for the Allylation of Compound 6 with Potassium Allyltrifluoroborate (2) Catalyzed by $18-C-6$}

To a solution of $6(150 \mathrm{mg}, 0.5 \mathrm{mmol})$ in $\mathrm{CH}_{2} \mathrm{Cl}_{2}(1.5 \mathrm{~mL})$ was added $18-C-6(13 \mathrm{mg}, 10 \mathrm{~mol} \%)$ followed by potassium allyltrifluoroborate $(2,89 \mathrm{mg}, 0.60 \mathrm{mmol})$ and water $(1.5 \mathrm{~mL})$. The biphasic mixture was stirred for 15 minutes, diluted with EtOAc $(15 \mathrm{~mL})$ and washed with a saturated solution of $\mathrm{K}_{2} \mathrm{CO}_{3}(3 \times 15 \mathrm{~mL})$. The combined organic layers were dried over $\mathrm{MgSO}_{4}$, filtered and the solvents were removed in vacuo to yield $7(138 \mathrm{mg}, 85 \%)$ without the need of further purification. 
(5S,7Z,9E)-5-(Methoxymethoxy)-11-(tetrahydro-2H-pyran-2-yloxy)undeca-1,7,9-trien-3-ol: ${ }^{1} \mathrm{H}-\mathrm{NMR}$ $\left(\mathrm{CDCl}_{3}\right) \delta 6.57-6.47(\mathrm{~m}, 1 \mathrm{H}), 6.11(\mathrm{td}, J=11.1,1.2 \mathrm{~Hz}, 1 \mathrm{H}), 5.90-5.75(\mathrm{~m}, 2 \mathrm{H}), 5.44(\mathrm{dd}, J=18.6$, $7.5 \mathrm{~Hz}, 1 \mathrm{H}), 5.14-5.07(\mathrm{~m}, 3 \mathrm{H}), 4.73(\mathrm{dd}, J=15.6,7.5 \mathrm{~Hz}, 2 \mathrm{H}), 4.66-4.63$ (m, 2H), 4.30 (dd; $J=12.9$, $6.0 \mathrm{~Hz}, 1 \mathrm{H}), 4.03(\mathrm{dd}, J=12.9,6.5 \mathrm{~Hz}, 1 \mathrm{H}), 3.95-3.78(\mathrm{~m}, 3 \mathrm{H}), 3.55-3.46(\mathrm{~m}, 1 \mathrm{H}), 3.39(\mathrm{~s}, 3 \mathrm{H})$, 2.50-2.41 (m, 1H), $2.23(\mathrm{t}, J=6.6 \mathrm{~Hz}, 3 \mathrm{H}) 1.88-1.48(\mathrm{~m}, 7 \mathrm{H}) ;{ }^{13} \mathrm{C}-\mathrm{NMR}\left(\mathrm{CDCl}_{3}\right) \delta 134.89,130.25$, $127.57,127.22$, 126.81, 117.53, 97.88, 96.32, 95.22, 75.31, 67.38, 67;09, 62.15, 55.79, 41.98, 40.97, $30.57,25.40,19.41$.

\section{Conclusions}

In summary, we have demonstrated that a catalytic amount of $18-C-6$ can efficiently promote the allylation of aldehydes. This green method features the use of small catalyst loads, avoid the preparation of unstable allyl organometallics and the products were obtained in short reaction times with high yield and purity at room temperature. The approach is complementary to the previously described methods for the synthesis of homoallylic alcohols and should be synthetically useful for the synthesis of more complex compounds.

\section{Supplementary Materials}

Supplementary materials can be accessed at: http:/www.mdpi.com/1420-3049/17/12/14099/s1.

\section{Acknowledgments}

The authors gratefully acknowledge FACEPE (APQ-1402-1.06/10), CNPq (484778/2011-0), CAPES and INCT-INAMI for financial support. P.H.M and J.C.R.F. are thankful to CNPq for their fellowships.

\section{References}

1. Denmark, S.E.; Fu, J. Catalytic Enantioselective Addition of Allylic Organometallic Reagents to Aldehydes and Ketones. Chem. Rev. 2003, 103, 2763-2793.

2. Yamamoto, Y.; Asao, N. Selective reactions using allylic metals. Chem. Chem. Rev. 1993, 93, 2207.

3. Johnson, A.A.; Sharpless, K.B. Catalytic asymmetric epoxidation of allylic alcohols. In Comprehensive Organic Synthesis; Pergamon Press: Oxford, UK, 1990; Volume 7.

4. Luche, J.L.; Damiano, J.C. Ultrasounds in organic syntheses. 1. Effect on the formation of lithium organometallic reagents. J. Am. Chem. Soc. 1980, 102, 7926-7927.

5. Zhang, W.-C.; Li, C.-J. Magnesium-Mediated Carbon-Carbon Bond Formation in Aqueous Media: Barbier-Grignard Allylation and Pinacol Coupling of Aldehydes. J. Org. Chem. 1999, 64, 3230-3236.

6. Barczak, N.T.; Jarvo, E.R. Silver-Catalyzed, Manganese-Mediated Allylation and Benzylation Reactions of Aldehydes and Ketones. Eur. J. Org. Chem. 2008, 5507-5510.

7. Petrier, C.; Luche, J.L. Allylzinc reagents additions in aqueous media. J. Org. Chem. 1985, 50, 910-912.

8. Zhou, J.-Y.; Sun, G.-F.; Zhang, M.-F.; Jia, Y.; Wu, S.-H. Lead-promoted Barbier-type reaction of aldehydes with propargyl bromides in aqueous media. Chin. J. Chem. 1997, 15, 361-365. 
9. Zhang, X.; Qiu, R.; Tan, N.; Yin, S.; Xia, J.; Luo, S.; Au, C.-T. Air-stable hypervalent organobismuth(III) tetrafluoroborate as effective and reusable catalyst for the allylation of aldehyde with tetraallyltin. Tetrahedron Lett. 2010, 51, 153-156.

10. Iamamoto, T.; Kusumoto, T.; Tawarayama, Y.; Sugiura, Y.; Mit, T. Carbon-carbon bond-forming reactions using cerium metal or organocerium(III) reagents. J. Org. Chem. 1984, 49, 3904-3912.

11. Suzuki, T.; Atsumi, J.-I.; Sengoku, T.; Takahashi, M.; Yoda, H. Indium-catalyzed enantioselective allylation of aldehydes with $\beta$-carbonyl allylstannanes: An efficient synthetic method for chiral $\alpha$-methylene- $\gamma$-lactones. J. Organomet. Chem. 2010, 695, 128-136.

12. Guimarães, R.L.; Lima, D.J.P.; Barros, M.E.S.B.; Cavalcanti, L.N.; Hallwass, F.; Navarro, M.; Bieber, L.W.; Malvestiti, I. Aqueous Barbier Allylation of Aldehydes Mediated by Tin. Molecules 2007, 12, 2089-2105.

13. Jiang, G.; List, B. Acid-Catalyzed $\alpha$-Allylation of Aldehydes with Allylic Alcohols. Adv. Synth. Catal. 2011, 353, 1667-1670.

14. Bhuyan, B.K.; Borah, A.J.; Senapati, K.K.; Phukan, P. Ti-exchanged ZSM-5 as heterogeneous catalyst for allylation of aldehydes with allyltributylstannane. Tetrahedron Lett. 2011, 52, 2649-2651.

15. Alper, H.; Vasylyev, M. Rhodium-Catalyzed Reductive Allylation of Conjugated Aldehydes with Allyl Acetate. J. Org. Chem. 2010, 75, 2710-2713.

16. Deng, D.; Liu, B.; Wang, L.; Fu, W. $\left[\mathrm{Cd}_{2}(\operatorname{tren})_{2}(d l\right.$-alaninato $\left.)\right]\left(\mathrm{ClO}_{4}\right)_{3}$ : an efficient water-compatible Lewis acid catalyst for chemo-, regio-, and diastereo-selective allylation of various aldehydes. Tetrahedron Lett. 2010, 51, 5567-5570.

17. Bian, Y.-J.; Zhao, H.-M.; Yu, X.-G. Allylation Reactions of Aromatic Aldehydes with Antimony in Aqueous Media Under Ultrasonic Irradiation. Synth. Commun. 2009, 39, 2370-2377.

18. Shimizu, H.; Igarashi, T.; Miura, T.; Murakami, M. Rhodium-Catalyzed Reaction of 1-Alkenylboronates with Aldehydes Leading to Allylation Products. Angew. Chem. Int. Ed. 2011, 50, 11465-11469.

19. Ramadhar, T.R.; Batey, R.A. Allylation of Imines and Their Derivatives with Organoboron Reagents: Stereocontrolled Synthesis of Homoallylic Amines. Synthesis 2011, 1321-1346.

20. Kennedy, J.W.J.; Hall, D.G. Recent Advances in the Activation of Boron and Silicon Reagents for Stereocontrolled Allylation Reactions. Angew. Chem. Int. Ed. 2003, 42, 4732 - 4739.

21. Massa, A.; Acocella, M.R.; De Sio, V.; Villano, R.; Scettri, A. A catalytic asymmetric allylation of aldehydes with allyl trichlorosilane activated by a chiral tetradentate bis-sulfoxide. Tetrahedron: Asymmetry 2009, 20, 202-204.

22. Souza, R.F.M.; Areias, M.C.C.; Bieber, L.W.; Navarro, M. Electrochemical allylation of aldehydes in a solvent-free cavity cell with a graphite powder cathode. Green Chem. 2011, 13, 1118.

23. Preite, M.D.; Jorquera-Geroldi, H.A.; Pérez-Carvajal, A. Barbier allylation of aldehydes and ketones with aluminium and catalytic indium metal: an economical alternative. ARKIVOC 2011, 7, 380-388.

24. Dam, J.H.; Fristrup, P.; Madsen, R. Combined Experimental and Theoretical Mechanistic Investigation of the Barbier Allylation in Aqueous Media. J. Org. Chem. 2008, 73, 3228-3235.

25. Oliveira, R.A. Organotrifluoroborate Salts. Synlett 2009, 505-506.

26. Darses, S.; Genet, J.-P. Potassium Organotrifluoroborates: New Perspectives in Organic Synthesis. Chem. Rev. 2008, 108, 288. 
27. Stefani, H.A.; Cella, R.; Vieira, A.S. Recent advances in organotrifluoroborates chemistry. Tetrahedron 2007, 63, 3623-3658.

28. Molander, G.A.; Figueroa, R. Organotrifluoroborates: Expanding organoboron chemistry. Aldrichim. Acta 2005, 38, 49-56.

29. Nowrouzi, F.; Thadani, A.N.; Batey, R.A. Allylation and Crotylation of Ketones and Aldehydes Using Potassium Organotrifluoroborate Salts under Lewis Acid and Montmorillonite K10 Catalyzed Conditions. Org. Lett. 2009, 11, 2631-2634.

30. Matsuoka, H.; Kondo, K. General and convenient TsOH-induced allylboration of ketones. Tetrahedron Lett. 2009, 50, 2320-2321.

31. Thadani, A.N.; Batey, R.A. A Mild Protocol for Allylation and Highly Diastereoselective Syn or Anti Crotylation of Aldehydes in Biphasic and Aqueous Media Utilizing Potassium Allyl- and Crotyltrifluoroborates. Org. Lett. 2002, 4, 3827-3830.

32. Nakamura, H.; Shimizu, K. Catalytic reactions of bis- $\pi$-allylpalladium generated from allyltrifluoroborate. Tetrahedron Lett. 2011, 52, 426-429.

33. Shaghafi, M.B.; Kohn, B.L.; Jarvo, E.R. Palladium-Catalyzed Conjugate Allylation Reactions of $\alpha, \beta$-Unsaturated N-Acylpyrroles. Org. Lett. 2008, 10, 4743-4746.

34. Batey, R.A.; Thadani, A.N.; Smil, D.V. Potassium allyl- and crotyltrifluoroborates: Stable and efficient agents for allylation and crotylation. Tetrahedron Lett. 1999, 40, 4289-4292.

35. Horváth, I.T.; Anatas, P.T. Innovations and Green Chemistry. Chem. Rev. 2007, 107, 2169-2173.

36. Hutchings, G.J. A golden future for green chemistry. Catal. Today 2007, 122, 196-200.

37. Hancock, R.D.; Martell, A.E. Ligand design for selective complexation of metal ions in aqueous solution. Chem. Rev. 1989, 89, 1875-1914.

38. Bloch, R. Additions of Organometallic Reagents to $\mathrm{C}=\mathrm{N}$ Bonds: Reactivity and Selectivity. Chem. Rev. 1998, 98, 1407-1438.

39. Rüdel, H. Case study: Bioavailability of tin and tin compounds. Ecotoxicol. Environ. Saf. 2003, $56,180-189$.

40. Oliveira, R.A.; Silva, R.O.; Molander, G.A.; Menezes, P.H. ${ }^{1} \mathrm{H},{ }^{13} \mathrm{C},{ }^{19} \mathrm{~F}$ and ${ }^{11} \mathrm{~B}$ NMR spectral reference data of some potassium organotrifluoroborates. Magn. Res. Chem. 2009, 47, 873-878.

41. Petrillo, D.E.; Kohli, R.K.; Molander, G.A. Accurate mass determination of organotrifluoroborates. J. Am. Soc. Mass Spectrom. 2007, 18, 404-405.

42. Oliveira, R.A.; Savegnago, L.; Jesse, C.R.; Menezes P.H.; Molander G.A.; Nogueira, C.W. Toxicological investigation and antinociceptive property of potassium thiophene-3trifluoroborate. Basic Clin. Pharmacol. Toxicol. 2009, 104, 448-454.

43. Jaruchoktaweechai, C.; Suwanborirux, K.; Tanasupawatt, S.; Kittakoop, P.; Menasveta, P. New Macrolactins from a Marine Bacillus sp. Sc026. J. Nat. Prod. 2000, 63, 984-986.

44. Oliveira, R.A.; Oliveira, J.M.; Rahmeier, L.H.S.; Comasseto, J.V.; Marino, J.P.; Menezes, P.H. Synthesis of the C7-C24 fragment of (-)-Macrolactin F. Tetrahedron Lett. 2008, 49, 5759-5761.

45. Molander, G.A.; Figueroa, R. cis-Dihydroxylation of Unsaturated Potassium Alkyl- and Aryltrifluoroborates. Org. Lett. 2006, 8, 75-78.

46. Denmark, S.E.; Nguyen, S.T. Catalytic, Nucleophilic Allylation of Aldehydes with Allyl Acetate. Org. Lett. 2009, 11, 781-784. 
47. Li, G.-L.; Zhao, G. Allylation of Aldehydes and Imines: Promoted by Reuseable Polymer-Supported Sulfonamide of N-Glycine. Org. Lett. 2006, 8, 633-636.

Sample Availability: Samples of the compounds $\mathbf{2}, \mathbf{3 a}-\mathbf{0}, \mathbf{4}-\mathbf{7}$ are available from the authors.

(C) 2012 by the authors; licensee MDPI, Basel, Switzerland. This article is an open access article distributed under the terms and conditions of the Creative Commons Attribution license (http://creativecommons.org/licenses/by/3.0/). 